\title{
Criterios de Interpretación para California Mastitis Test en el Diagnóstico de Mastitis Subclínica en Bovinos
}

\author{
Interpretation Criteria for California Mastitis Test in the Diagnosis of \\ Subclinical Mastitis in Cattle
} Oscar Elisban Gómez-Quispe ${ }^{1,3}$, Crhis Stefani Santivañez-Ballón'², Fernando Arauco-
Villar $^{3}$, Oscar Henry Espezua-Flores ${ }^{4}$, Jorge Manrique-Meza

\section{Resumen}

\begin{abstract}
El estudio se realizó en el distrito de Tamburco (Abancay, Apurímac, Perú), con el objetivo de comparar la interpretación de los resultados de la prueba «California Mastitis Test» (CMT) en el diagnóstico de la mastitis subclínica en bovinos. Se trabajó con 209 vacas de una población de 459 en ordeño manual, pertenecientes a 95 criadores, donde se evaluaron 828 cuartos mamarios funcionales y se estimaron los principales criterios de interpretación de la CMT. La prevalencia de mastitis subclínica fue de 72.3 y $65.6 \%$ en las vacas y de 48.7 y $42.3 \%$ en el total de cuartos mamarios, considerando y sin considerar trazas como positivos, respectivamente, y sin diferencia significativa. La prevalencia de mastitis subclínica en los cuartos mamarios individuales varió entre 48.3 y $49.3 \%$ y de 40.0 y $45.5 \%$, según el cuarto, considerando y sin considerar trazas, respectivamente $(\mathrm{p}<0.05)$. Se encontró una mayor intensidad de reacción positiva en los grados de 1, 2 y 3 cruces y menor en el grado trazas. El índice de mastitis subclínica (IMSC) fue 1.30 y el índice de bajo riesgo - alto riesgo (I BR/AR) fue de 2.71. Se concluye que los criterios de interpretación de la CMT para mastitis subclínica tienen similares significados, pero cada uno de ellos proporciona detalles particulares de la enfermedad; asimismo, los indicadores IMSC e I BR/AR proveen información útil sobre la dinámica de la mastitis subclínica.
\end{abstract}

Palabras clave: RCS; ubre; CMT; vacas; prevalencia

\footnotetext{
${ }^{1}$ Facultad de Medicina Veterinaria y Zootecnia, Universidad Nacional Micaela Bastidas de Apurímac, Perú

${ }^{2}$ Fondo de Desarrollo Ganadero, Agrícola y Agroindustrial, Apurímac, Perú

${ }^{3}$ Facultad de Zootecnia de la Universidad Nacional del Centro del Perú, Huancayo, Perú

${ }^{4}$ Facultad de Medicina Veterinaria y Zootecnia, Universidad Nacional del Altiplano, Puno, Perú

${ }^{5}$ Laboratorio Veterinario del Sur - LABVETSUR, Perú

${ }^{6}$ E-mail: oegomezq@hotmail.com
}

Recibido: 30 de enero de 2014

Aceptado para publicación: 30 de agosto de 2014 
The study was conducted in the district of Tamburco (Abancay, Apurimac, Peru) with the aim of comparing interpretation criteria of California Mastitis Test (CMT) in the diagnosis of subclinical mastitis in cattle. Two hundred and nine cows from a population of 459 hand-milked cows belonging to 95 breeders were used. A total of 828 functional mammary quarters were evaluated and the main criteria for CMT interpretation were estimated. The prevalence of subclinical mastitis in cows was 72.3 and $65.6 \%$, and in total mammary quarters was 48.7 and $42.3 \%$ with and without considering traces as positives respectively, and without statistical difference. The estimated prevalence in individual mammary quarters varied between 48.3 to $49.3 \%$ and depending of the mammary quarter between 40.0 to $45.5 \%$ with and without considering traces as positives respectively $(\mathrm{p}<0.05)$. More intense positive reaction was found in grades 1, 2 and 3 crosses and lower in the trace grade. Subclinical Mastitis Index (IMSC) was 1.30 and the Low Risk - High Risk Index (I BR/AR) was 2.71. It was concluded that the CMT interpretation criteria for subclinical mastitis have similar meanings, but each provides specific details of the disease; besides, the IMSC and I BR/HR indices provide useful information on the dynamics of subclinical mastitis.

Key words: SCC; udder; CMT; cows; prevalence

\section{INTRODUCCIÓN}

Los valles interandinos del Perú son aptos para la crianza de ganado vacuno con fines de comercialización de leche; sin embargo, uno de los problemas que afecta la ganadería lechera es la ocurrencia de mastitis en las vacas en ordeño. La mastitis es un grave problema que afecta la industria lechera a nivel mundial (Fetrow et al., 1991). Implica grandes pérdidas económicas debido a la disminución de la producción de leche, aumento del número de vacas descartadas, gastos médicos y desecho de leche de animales tratados (Searcy et al., 1995).

En condiciones de campo, una gran cantidad de animales afectados con mastitis pasan desapercibidos en la evaluación clínica (Patiño, 2008), pero podrían ser diagnosticados mediante pruebas de laboratorio y de campo como el California Mastitis Test (CMT) (AL-Edany et al., 2012). Este es un método ampliamente utilizado, dada su utilidad práctica, bajo costo y rápido resultado (Sanford et al., 2006). Los valores del CMT están relacionados con el número resultante del recuento de células somáticas (RCS) en la leche de vacas con cuartos mamarios afectados. En el caso de mastitis subclínicas, las células somáticas (CS) migran de la sangre hacia la leche como respuesta a la infección, aumentando con la severidad del proceso (Philpot y Nickerson, 2000).

El análisis de los resultados mediante la prueba de campo CMT, según la literatura científica, no es uniforme. Algunos investigadores consideran como vaca afectada no solo aquellas con grados de reacción positivos (Novoa, 2003; Roy et al., 2009; Coronel et al., 2011; Zambrano et al., 2011), sino, además, con la presencia de trazas, grado que corresponde a un RCS de 200000 a 400 000/ml (Ruiz et al., 2011; Solís, 2007; Ramírez et al., 2011).

La estimación de la prevalencia de mastitis mediante el CMT se calcula de varias formas: prevalencia total en vacas con al menos un cuarto mamario afectado $(\mathrm{P})$, 
prevalencia en el total de cuartos mamarios (PTC), prevalencia en los cuartos mamarios individuales (PCI), proporción de cuartos mamarios afectados (PCA) e intensidad de reacción (IR), entre otros indicadores. En todos los casos, se considera la variante de considerar el grado trazas como positivo o negativo. En este contexto, considerar como positivo a mastitis subclínica al grado de reacción trazas del CMT, indicado como mastitis subclínica leve por varios investigadores (Philpot y Nickerson, 2000; Andresen, 2001; Sharif y Muhammad, 2008), permitiría realizar un adecuado control de la enfermedad y tendría un posible impacto favorable en el monitoreo de programas preventivos y de salud epidemiológica.

En otros estudios, los resultados del CMT son interpretados mediante el índice de mastitis subclínica (IMSC) (Alonso, 1979; Delgado, 1983; Arana, 1992; Romaní, 1995; Castillo et al., 2007), que mide el nivel de recuento celular o mastitis subclínica de una explotación, y con el índice de bajo riesgo alto riesgo (I BR/AR) que evalúa la relación entre el RCS de alto riesgo $[(++)+(+++)]$ con respecto a bajo riesgo $[\mathrm{N}+\mathrm{T}+(+)]$, como herramientas para el monitoreo de la dinámica epidemiológica de la mastitis subclínica en el tiempo, en establos con altos niveles de prevalencia.

Estas consideraciones hacen que se manejen diversas interpretaciones de la información disponible; de allí que el objetivo de la presente investigación fue comparar los criterios de interpretación de los resultados de CMT en el diagnóstico de mastitis subclínica bovina.

\section{Materiales y Métodos}

\section{Localización y Muestras}

El estudio se llevó a cabo en el distrito de Tamburco (Kerapata), provincia de Abancay, departamento de Apurímac (Perú), en el último semestre de 2012. La zona está ubicada entre 2700 y $3100 \mathrm{~m}$ de altitud, con una temperatura promedio anual máxima y mínima de 18 y $1.3{ }^{\circ} \mathrm{C}$, humedad relativa entre 59 y $70 \%$ y precipitación pluvial entre 641 y $1119 \mathrm{~mm} / \mathrm{año}$.

En la mayoría de las fincas del estudio, se utilizaba el sistema de pastoreo a estaca, con alimentación a base de Pennisetum clandestinum, Trifolium sp, Medicago sativa y Zea mays y suplementación (concentrado casero). La producción promedio de leche fue $10.8 \mathrm{~kg} / \mathrm{vaca} /$ día, con ordeño manual, dos veces por día y con presencia de ternero. Las fincas tenían cinco bovinos en promedio, donde usualmente dos vacas estaban en producción. La composición racial de la población fue 54, 33, 5 y $8 \%$ para Holstein, Criollo, Brown Swiss y cruces, respectivamente.

El estudio fue de tipo transversal, con un tamaño de población de 459 vacas en producción. Se utilizó una muestra aleatoria de 209 vacas ( $\mathrm{p}=0.6$; nivel de confianza de $95 \%$ ) de 95 criadores, distribuyéndose por conglomerados, según la metodología propuesta por Mateu y Casal (2003) para muestreos en epidemiología.

\section{Diagnóstico de Mastitis Subclínica}

La prueba de CMT se realizó antes del ordeño del animal, tomándose una muestra aproximada de $2 \mathrm{ml}$, después de eliminarse los primeros chorros de leche, siguiendo el procedimiento propuesto por Schalm y Noorlander (1957) y el US National Mastitis Council (Philpot y Nickerson, 2000).

\section{Procesamiento y Análisis de Datos}

La prevalencia de mastitis subclínica mediante la prueba de CMT se determinó con y sin la presencia de trazas (RCS de 200 a $400 \mathrm{mil} / \mathrm{ml}$ ). No se tomaron en cuenta los cuartos ciegos (no funcionales). 
Los datos se procesaron de acuerdo a las fórmulas epidemiológicas siguientes:

- Prevalencia en vacas $(\mathrm{P})=\left(\mathrm{N} .^{\circ}\right.$ de vacas positivas / N. ${ }^{\circ}$ total de vacas $) \times 100$

- Prevalencia en el total de cuartos mamarios $(\mathrm{PTC})=\left(\mathrm{N} .^{\circ}\right.$ de cuartos positivos / N. ${ }^{\circ}$ total de cuartos) $\times 100$

- Prevalencia en los cuartos mamarios individuales $(\mathrm{PCI})=\left(\mathrm{N} .^{\circ}\right.$ de cuartos positivos por posición / N. ${ }^{\circ}$ total de cuartos por posición) $\times 100$

- Proporción de cuartos mamarios afectados $(\mathrm{PCA})=\left(\mathrm{N} .^{\circ}\right.$ de cuartos positivos por posición / N. ${ }^{\circ}$ total de cuartos positivos) $\times 100$

- Intensidad de reacción $(\mathrm{IR})=\left(\mathrm{N} .^{\circ}\right.$ de casos por grado de reacción / N. ${ }^{\circ}$ total de casos o $\mathrm{N} .^{\circ}$ total de cuartos) $\times 100$

En el cálculo de la prevalencia de vacas, se entiende por vaca positiva cuando al menos tiene un cuarto afectado.

Para efectos de diagnóstico, en la determinación de la prevalencia en vacas, prevalencia en el total de cuartos mamarios, prevalencia en los cuartos mamarios individuales y en la estimación de la proporción de cuartos mamarios afectados, se consideran las variantes de indicar al grado trazas como positivos al CMT o como negativo; y en la intensidad de reacción, se considera la cantidad de cuartos mamarios afectados en cada grado de reacción (trazas, una, dos y tres cruces).

Para determinar el índice de mastitis subclínica (IMSC) y el índice de bajo riesgo alto riesgo (I BR/AR) se utilizaron las fórmulas propuestas por Alonso (1979), utilizadas como herramientas de evaluación dinámica de la mastitis en su forma subclínica (Delgado, 1983; Arana, 1992; Romaní, 1995; Castillo et al., 2007). Así, IMSC $=((\mathrm{N} \times 0)+$ $(\mathrm{T} \times 1)+[(+) \times 2]+[(++) \times 3]+[(+++) \times 4]$ $/ \mathrm{N} .^{\circ}$ pezones funcionales $) \times 100$; donde $\mathrm{N}$ $($ negativo $)=0, \mathrm{~T}($ trazas $)=1,+=2,++=3$, $+++=4$, siendo,,++++++ los grados de reacción a CMT. Asimismo, el Índice BR/AR
$=((\mathrm{N})+(\mathrm{T})+(+) /(++)+(+++)) ;$ donde $\mathrm{N}$ $=$ negativo, $\mathrm{T}=$ trazas,$+=$ prevalencia débil, $++=$ prevalencia evidente, $+++=$ prevalencia fuerte.

Para analizar las diferencias en las prevalencias, reacción positiva de los cuartos y la IR de los grados de la CMT, se utilizó la prueba de comparación de proporciones empleando el programa SPSS v. 9.0 para Windows (SPSS Inc, Chicago IL, USA).

\section{Resultados y Discusión}

\section{Prevalencia de Mastitis Subclínica}

La prevalencia de mastitis subclínica en vacas fue $72.3 \%$ cuando se considera al grado trazas como positivos y de $65.6 \%$ en caso contrario, sin diferencia estadística entre ambos criterios de interpretación (Cuadro 1). La prevalencia de mastitis subclínica en vacas, considerando el grado trazas como positivos, fue superior con respecto a reportes en el Perú (Cuayla, 2010; Coronel et al., 2011) y en el extranjero (Novoa, 2003). Asimismo, fue superior a otros resultados, cuando se excluyeron las trazas como positivos, tanto en América Latina (Torrez y Duartes, 2006; Solís, 2007; Acuña y Rivadeneira, 2008; Ramírez et al., 2011; Ruiz et al., 2011) como en otras latitudes (Roy et al., 2009).

La prevalencia de mastitis subclínica en el total de cuartos mamarios fue 48.7 y $42.3 \%$ en diagnósticos con y sin trazas, respectivamente ( $\mathrm{p}<0.05$ ) (Cuadro 1). El 48.7\% de prevalencia considerando el grado trazas como positivo fue superior al reporte de Pastor y Bedolla (2008) en México, pero menor que otros reportes en el país (Coronel et al., 2011). En el caso de la prevalencia sin considerar el grado trazas como positivo, el resultado fue similar (Torrez y Duartes, 2006; Solís, 2007) o superior a otros estudios (Roy et al., 2009; Cuayla, 2010; Ramírez et al., 2011). La disparidad de resultados se puede atribuir a factores intrínsecos de cada ámbito que 
Cuadro 1. Prevalencia de mastitis subclínica en vacas (con al menos un cuarto mamario afectado), en el total de cuartos mamarios, y en cuartos mamarios individuales en vacas del distrito de Tamburco, Abancay, Apurímac, Perú (2012)

\begin{tabular}{|c|c|c|c|c|c|c|}
\hline \multirow{2}{*}{$\begin{array}{c}\text { Prevalencia } \\
\quad(\%)\end{array}$} & \multicolumn{3}{|c|}{ Con trazas } & \multicolumn{3}{|c|}{ Sin trazas } \\
\hline & (Casos/n) & $\%$ & $\mathrm{IC}_{95}$ & (Casos/n) & $\%$ & $\mathrm{IC}_{95}$ \\
\hline \multicolumn{7}{|l|}{ Global } \\
\hline Vacas & $(151 / 209)$ & $72.3^{\mathrm{a}}$ & 66.278 .3 & $(137 / 209)$ & $65.6^{\mathrm{a}}$ & 59.172 .0 \\
\hline Total de cuartos mamarios & $(403 / 828)$ & $48.7^{\mathrm{a}}$ & 45.352 .1 & $(350 / 828)$ & $42.3^{\mathrm{a}}$ & 38.945 .6 \\
\hline \multicolumn{7}{|l|}{ Cuartos mamarios individuales } \\
\hline Posición Ant. izquierdo & $(101 / 209)$ & $48.3^{\mathrm{a}}$ & 41.555 .1 & $(88 / 209)$ & $42.1^{\mathrm{b}}$ & 33.346 .7 \\
\hline Posición Ant. derecho & $(101 / 207)$ & $48.8^{\mathrm{a}}$ & 42.055 .6 & $(86 / 207)$ & $41.6^{\mathrm{b}}$ & $34.8 \quad 48.3$ \\
\hline Posición Post. izquierdo & $(099 / 205)$ & $48.3^{\mathrm{a}}$ & 41.655 .1 & $(82 / 205)$ & $40.0^{\mathrm{c}}$ & $35.4 \quad 48.8$ \\
\hline Posición Post. derecho & $(102 / 207)$ & $49.3^{\mathrm{a}}$ & $42.5 \quad 56.1$ & $(94 / 207)$ & $45.4^{\mathrm{a}}$ & 38.652 .2 \\
\hline
\end{tabular}

a,b,c Superíndices diferentes dentro de filas en el análisis global o dentro de columnas en el análisis por cuartos mamarios indican diferencia estadística $(p<0.05)$

$\mathrm{IC}_{95}=$ Intervalo de confianza al $95 \%$

condicionan la presentación y forma de manifestación de la enfermedad y al criterio empleado en la estimación e interpretación de la prevalencia de la mastitis subclínica para el total de cuartos mamarios.

En diversos estudios sobre prevalencia de mastitis subclínica se consideran las trazas como casos positivos (Novoa, 2003; Cuayla, 2010; Coronel et al., 2011) y en otros estudios como casos negativos (Torrez y Duartes, 2006; Solís, 2007; Acuña y Rivadeneira, 2008; Roy et al., 2009; Ramírez et al., 2011; Ruiz et al., 2011). Del mismo modo, la determinación de la prevalencia en el total de cuartos se hace tanto incluyendo el grado de reacción trazas (Torrez y Duartes, 2006; Solís, 2007; Pastor y Bedolla, 2008; Coronel et al., 2011; Ramírez et al., 2011) como excluyéndolo (Torrez y Duartes, 2006; Solís, 2007; Roy et al., 2009; Cuayla, 2010; Ramírez et al., 2011).

En este contexto, la Norma Técnica Peruana 202.001:2010 (INDECOPI, 2010) sobre leche cruda, indica que un conteo de células somáticas hasta 500 000/ml es de calidad higiénica aceptable. Aunque esta norma está referida al conteo de CS en leche de tanque y en condiciones de ordeño mecánico, estaría considerando al grado de reacción trazas (de 200 a $400 \mathrm{mil} \mathrm{CS} / \mathrm{ml}$ ) como normal y aceptable. Para muchos investigadores, un RCS superior a 200 000/ml es indicativo de mastitis subclínica (Jones, 1986; NMC, 1996; Philpot y Nickerson, 2000; Sharif y Muhammad, 2008); en tanto que RCS mayores a 400 000/ml estaría implicando elevadas tasas de infección (Jones et al., 1984), causando una disminución de los potenciales ingresos económicos por venta de leche (Philpot y Nickerson, 2000). Estas consideraciones las confirma Brito et al. (1999), al aislar patógenos primarios ( $S$. aureus e $S$. agalactiae) y secundarios (Corynebacterium sp y Staphylococcus sp) de cuartos mamarios de vacas con reacción negativa y grado trazas al CMT. Es posible que la inclusión del grado trazas de la CMT considerado como mastitis subclínica leve (Philpot y 
Nickerson, 2000; Andresen, 2001), en la estimación de la prevalencia con fines de diagnóstico, facilitaría un adecuado seguimiento y control de la enfermedad. Asimismo, sería conveniente revisar la Norma Técnica Peruana 202.001:2010 a fin de promover el control de la mastitis subclínica en las fincas rurales.

En cuanto a los cuartos mamarios individuales, las prevalencias que consideraron al grado trazas como positivos estuvieron entre 48.3 y $49.3 \%$, sin diferencias estadísticas entre ellos (Cuadro 1); estos valores fueron inferiores a los reportados por Flores y García (2005). Por otro lado, al excluir las trazas de la estimación, el cuarto posterior izquierdo fue el menos afectado $(40.0 \%)$ y el cuarto posterior derecho fue el más afectado (45.4\%), habiendo diferencias significativas entre cuartos $(\mathrm{p}<0.05)$. Al respecto, existen estudios que demuestran que los cuartos anteriores son más propensos a presentar una mayor frecuencia de reacciones positivas, especialmente el cuarto anterior derecho (Duarte, 2004; Torrez y Duartes, 2006; Solís, 2007); aunque Ferraror (1992) indica que los cuartos posteriores presentan mayores niveles de infección. Estas diferencias con los resultados del presente estudio podrían deberse al ordeño manual (Ferraror, 1992).

Los resultados obtenidos tendrían el mismo valor que la «prevalencia por total de cuartos mamarios»; sin embargo, el análisis por cuartos mamarios facilitaría una mejor información acerca de la mayor o menor afectación de los cuartos según posición en la ubre, siendo de mayor utilidad para la toma de decisiones en un programa preventivo y de salud epidemiológica de la mastitis subclínica en las fincas rurales.

\section{Proporción de Cuartos Mamarios Afec- tados}

La proporción de cuartos mamarios con reacción positiva fue cercana al $25 \%$, sin diferencia estadística entre cuartos, sea tomando en cuenta o no las trazas (Cuadro 2). Es- tos resultados fueron similares a los encontrados por Pastor y Bedolla (2008), quienes reportaron una distribución proporcional similar al presente estudio en condiciones de ordeño manual; sin embargo, Coronel et al. (2011) reportaron al cuarto anterior izquierdo como el más afectado en vacas cebú en la selva peruana.

\section{Intensidad de Reacción de los Grados de CMT}

Las reacciones positivas de los grados 1,2 y 3 cruces suman $42.25 \%$ y $49.7 \%$ incluyendo trazas; similar a lo obtenido en la «prevalencia en el total de cuartos mamarios». Asimismo, se encontraron mayores reacciones positivas en los grados 1,2 y 3 , sin diferencias estadísticas entre ellos, pero siendo superiores a la intensidad del grado trazas (Cuadro 3). Estos resultados concuerdan con los trabajos de Torrez y Duartes (2006), Solís (2007), Cuayla (2010) y Ramírez et al. (2011); no obstante, Pastor y Bedolla (2008) obtuvieron una mayor presentación del grado 3 , con respecto a los demás grados.

\section{IMSC e I BR/AR}

Según Romaní (1995), es factible utilizar el IMSC e I BR/AR en la evaluación de mastitis subclínica en términos de tiempo y población. Si la evaluación del CMT se realiza en forma periódica en zonas o fincas con alta prevalencia de mastitis subclínica, el empleo del IMSC e I BR/AR permitiría una interpretación más dinámica. Con el uso de estos indicadores, se toma en cuenta a todas las vacas que están en lactación, permitiendo una vigilancia constante de la sanidad de la ubre (Arauco y Mayorga, 2006).

El IMSC considerado como deseable es de 0.5. Mientras menor sea este índice, menor será la magnitud de la mastitis. Los resultados con la prueba de CMT a base de 828 cuartos funcionales (Cuadro 3) dan un IMSC de 1.30, que indicaría la presencia de 
Cuadro 2. Proporción (\%) de cuartos mamarios afectados con mastitis subclínica en 209 vacas del distrito de Tamburco, Abancay, Apurímac, Perú (2012)

\begin{tabular}{lllllll}
\hline \multirow{2}{*}{$\begin{array}{c}\text { Cuartos mamarios } \\
\text { Individuales }\end{array}$} & \multicolumn{3}{c}{ Con trazas } & \multicolumn{3}{c}{ Sin trazas } \\
\cline { 2 - 7 } & $($ Casos/n) $\%$ & $\mathrm{IC}_{95}$ & $($ Casos/n) $\%$ & $\mathrm{IC}_{95}$ \\
\hline Posición Ant. izquierdo & $(101 / 403) 25.1^{\mathrm{a}}$ & 20.8 & 29.3 & $(88 / 350) 25.1^{\mathrm{a}}$ & 20.1 & 29.1 \\
Posición Ant. derecho & $(101 / 403) 25.1^{\mathrm{a}}$ & 20.8 & 29.3 & $(86 / 350) 24.6^{\mathrm{a}}$ & 20.6 & 29.7 \\
Posición Post. izquierdo & $(099 / 403) 24.6^{\mathrm{a}}$ & 21.1 & 29.6 & $(82 / 350) 23.4^{\mathrm{a}}$ & 22.2 & 31.5 \\
Posición Post. derecho & $(102 / 403) 25.3^{\mathrm{a}}$ & 20.4 & 28.8 & $(94 / 350) 26.9^{\mathrm{a}}$ & 19.0 & 27.9 \\
\hline
\end{tabular}

${ }^{a}$ Superíndices similares indican ausencia de diferencia estadística

$\mathrm{IC}_{95}=$ Intervalo de confianza al $95 \%$

Cuadro 3. Intensidad de reacción de los grados de la CMT en mastitis subclínica bovina en el distrito de Tamburco, Abancay, Apurímac, Perú (2012)

\begin{tabular}{|c|c|c|c|c|c|c|c|c|}
\hline \multirow{2}{*}{$\begin{array}{c}\text { Grado de reacción } \\
\text { al CMT }\end{array}$} & \multicolumn{2}{|c|}{$\begin{array}{c}\mathrm{RCS}^{1} \\
(\mathrm{x} 1000)\end{array}$} & \multicolumn{5}{|c|}{ Cuartos mamarios } & \multirow{2}{*}{$\begin{array}{l}\text { Intensidad } \\
\text { de } \\
\text { reacción } \\
(\%)\end{array}$} \\
\hline & Media $^{2}$ & Rango $^{3}$ & $\mathrm{AD}$ & AI & PI & PD & Total & \\
\hline $\mathrm{N}$ : Negativos & 100 & $0-200$ & 106 & 108 & 106 & 105 & 425 & $51.3^{\mathrm{a}}$ \\
\hline T: Trazas & 300 & $200-400$ & 15 & 13 & 17 & 8 & 53 & $6.4^{\mathrm{e}}$ \\
\hline $\begin{array}{l}\text { 1: Positivo débil } \\
(+)\end{array}$ & 900 & $400-1200$ & 31 & 32 & 30 & 35 & 128 & $15.45^{\mathrm{b}}$ \\
\hline $\begin{array}{l}\text { 2: Positivo } \\
\text { evidente }(++)\end{array}$ & 2700 & $1200-5000$ & 32 & 28 & 27 & 34 & 121 & $14.6^{\mathrm{bc}}$ \\
\hline $\begin{array}{l}\text { 3: Positivo fuerte } \\
(+++)\end{array}$ & 8100 & $>5000$ & 23 & 28 & 25 & 25 & 101 & $12.2^{\mathrm{bd}}$ \\
\hline Total & & & 209 & 209 & 209 & 209 & 828 & 100.0 \\
\hline
\end{tabular}

${ }^{1}$ Recuento de células somáticas según nivel de la prueba de CMT

${ }^{2}$ Medias del CMT (según Rice,1981; Philpot y Nickerson, 2000)

${ }^{3}$ Rango del CMT (según Radostitis et al., 2006)

${ }^{4} \mathrm{AD}=$ Anterior derecho, $\mathrm{Al}=$ Anterior izquierdo, $\mathrm{Pl}=$ Posterior izquierdo, $\mathrm{PD}=$ Posterior derecho

$a, b, c, d$ Superíndices diferentes dentro de columnas indican diferencia estadística $(p<0.05)$

un valor elevado de CS que podría deberse a posibles deficiencias en el control sanitario de los rebaños. Sin embargo, el valor del presente estudio fue inferior a los reportados en otros estudios (1.93 por Aguilar et al. [2014], 1.77 a 1.81 por Castillo et al. [2009], 2.31 por Ferraror [1992] y 2.51 por Arana [1992]).
El I BR/AR resultante de 2.71 indicaría que la cantidad de cuartos mamarios con bajo riesgo respecto al total de cuartos con alto riesgo es cercana a tres veces más, siendo un índice con bajo valor. Este indicador fue cercano al valor de 2.31 reportado por Ferraror (1992) y al de 1.90 de Arana (1992). 


\section{Conclusiones}

- Los criterios de interpretación de la CMT para mastitis subclínica bovina tienen similares significados; pero cada uno proporciona detalles particulares de la enfermedad.

- Los indicadores IMSC e I BR/AR proveen información útil sobre la dinámica de la mastitis subclínica.

\section{Literatura Citada}

1. Acuña VL, Rivadeneira A. 2008. Aislamiento, identificación y antibiograma de patógenos presentes en leche con mastitis en ganaderías bovinas de la provincia de Pichincha. Tesis de Ingeniero Agropecuario. Sangolquí, Ecuador: Escuela Politécnica del Ejército de Ecuador. $158 \mathrm{p}$.

2. Aguilar A, Bañuelos J, Pimienta E, Aguilar A, Torres P. 2014. Prevalencia de mastitis subclínica en la región Ciénega del Estado de Jalisco. Abanico Vet 4(1): 24-31.

3. AL-Edany AA, Khudor $M H, A L-$ Mousawi KS. 2012. Comparison of three indirect tests for the diagnosis of bovine subclinical mastitis caused by coagulase negative staphylococci with their susceptibility to seven antibiotics. Bas J Vet Res 11: 75-83.

4. Alonso FR. 1979. Prevalencia de mastitis subclínica bovina en la cuenca del Lago de Maracaibo. En: I Jornadas Nacionales sobre Ganadería de Doble Propósito, Machiques, Venezuela. 23 p.

5. Andresen H. 2001. Mastitis: prevención y control. Rev Inv Vet Perú 12(2): 55-64.

6. Arana M. 1992. Prevalencia de la mastitis subclínica en el establo de la Facultad de Medicina Veterinaria de la Universidad Nacional Mayor de San Marcos. Tesis de Médico Veterinario. Lima: Univ Nacional Mayor de San Marcos. 40 p.
7. Arauco F, Mayorga N. 2006. Monitoreo epidemiológico de la mastitis subclínica en ganado Brown Swiss de la EEA El Mantaro-UNCP. En: Anales XXIX Reunión Científica de la Asociación Peruana de Producción Animal. Huancayo, Perú: APPA.

8. Brito MAVP, Brito JRF, Ribeiro MT, Veiga VMO. 1999. Padrão de infecção intramamária em rebanhos leiteiros: exame de todos os quartos mamários de vacas em lactação. Arq Bras Med Vet Zootec 51: 129-135.

9. Castillo M, Suniaga J, Rojas G, Hernández J, Caamaño J, Urbina A, Tovar T. 2009. Estudio de la prevalencia de mastitis subclínica en la zona alta del estado Mérida. Agric Andina 16: 39-48.

10. Castillo M, Suniaga J, Rojas G, Hernández J. 2007. Prevalencia de mastitis subclínica en la zona alta del estado Mérida. Estudio preliminar. Agric Andina 13: 65-70.

11. Coronel O, Bazán M, López M, Flores M, Laymes A, Muñoz H. 2011. Diagnóstico bacteriológico y prevalencia de la mastitis en vacas cebú en selva alta Satipo. En: XXXIV Reunión Científica de la Asociación Peruana de Producción Animal. Trujillo, Perú: APPA.

12. Cuayla E. 2010. Prevalencia de mastitis subclínica bovina en las secciones C, D y E en establos inscritos en el comité zonal de productividad lechera, distrito de Majes, departamento de Arequipa-2009. Tesis de Médico Veterinario y Zootecnista. Arequipa, Perú: Universidad Católica de Santa María. 78 p.

13. Delgado A. 1983. Mastitis subclínica en ganado vacuno lechero estabulado. En: Anales VI Reunión Científica de la Asociación Peruana de Producción Animal. Chiclayo, Perú: APPA.

14. Duarte A. 2004. Prevalencia de mastitis subclínica en el ganado criollo Reina en la finca Santa Rosa de la UNA en épo- 
ca de verano. Tesis de Ingeniero Agrónomo Zootecnista. Managua, Nicaragua: Universidad Nacional Agraria. $61 \mathrm{p}$.

15. Ferraror L. 1992. Análisis de la prevalencia de mastitis subclínica en vacas lecheras en Venezuela mediante la prueba de California Mastitis Test (CMT) y bacteriología. Tesis de Profesor Agregado. Venezuela: Universidad Central de Venezuela. $79 \mathrm{p}$.

16. Fetrow J, Mann D, Butcher K, McDaniel B. 1991. Production losses from mastitis: Carry-over from the previous lactation. J Dairy Sci 74: 833839.

17. Flores C, García J. 2005. Utilización de la propolina en el control de la mastitis bovina en la finca el Carmen del municipio de Camoapa, departamento de Boaco. Tesis de Médico Veterinario. Nicaragua: Universidad Nacional Agraria. $63 \mathrm{p}$.

18. [INDECOPI] Instituto Nacional de Defensa del Consumidor y Propiedad Intelectual. 2010. Leche cruda: Requisitos de calidad físicos, químicos y microbiológicos. Lima: INDECOPI. Norma Técnica Peruana (NTP) 202.001: 2010. 12 p.

19. Jones GM, Pearson RE, Clabaugh GA, Heald CW. 1984. Relationship between somatic cell counts and milk production. J Dairy Sci 67: 1823-1831.

20. Jones GM. 1986. Reducing somatic cell counts: Meeting the 1986 challenge Impact on producer and processor. $\mathbf{J}$ Dairy Sci 69: 1699-1707.

21. Mateu E, Casal J. 2003. Tamaño de la muestra. Rev Epidem Med Prev 1: 8-14.

22. [NMC] National Mastitis Council. 1996. Current concepts of bovine mastitis. $4^{\text {th }}$ ed. Madison: US National Mastitis Council Inc. 64 p.

23. Novoa R. 2003. Evaluación epizootiológica y económica de la mastitis bovina en rebaños lecheros especializados de la provincia de Cienfuegos. Tesis de
Magíster. Habana, Cuba: Universidad Agraria de la Habana «Fructuoso Rodríguez Pérez». $116 \mathrm{p}$.

24. Pastor J, Bedolla J. 2008. Determinación de la prevalencia de mastitis bovina en el municipio de Tarímbaro, Michoacán, mediante la prueba de California. REDVET 9(10). [Internet]. Disponible en: http://www.veterinaria. org/revistas/redvet/n101008/101004.pdf

25. Patiño N. 2008. Resistencia a antimicrobianos del Staphylococcus aureus en vacas lecheras con mastitis subclínica de tres municipios del estado de Michoacán. Tesis de Médico Veterinario y Zootecnista. México: Universidad Michoacana de San Nicolás de Hidalgo. $38 \mathrm{p}$.

26. Philpot W, Nickerson S. 2000. Ganando la lucha contra la mastitis. Naperville, IL, USA: Wesfalia Surge. 192 p.

27. Radostitis M, Gay CC, Hinchcliff KW, Constable PD. 2006. Veterinary medicine: a textbook of the diseases of cattle, horses, sheep, pigs and goats. $10^{\text {th }} \mathrm{ed}$. Amsterdam: Saunders. 2065 p.

28. Ramírez N, Arroyave O, Cerón M, Jaramillo M, Cerón J, Palacio L. 2011. Factores asociados a mastitis en vacas de la microcuenca lechera del altiplano norte de Antioquia, Colombia. Rev Med Vet 22: 31-42.

29. Rice DN. 1981. G81-556 Using the California Mastitis Test (CMT) to detect subclinical mastitis. Historical materials from University of Nebraska-Lincoln Extension. Paper 483.

30. Romani C. 1995. Propuesta de manejo dinámico de la mastitis subclínica bovina. Tesis de Médico Veterinario. Perú: Universidad Nacional Pedro Ruiz Gallo. 31 p.

31. Roy JP, Tremblay DD, DesCôteaux L, Messier S, Scholl D, Bouchard E. 2009. Evaluation of the California Mastitis Test as a precalving treatment selection tool for Holstein heifers. Vet Microbiol 134:136-142. doi: 10.1016/ j.vetmic.2008.09.020 
32. Ruiz A, Ponce P, Gomes G, Mota R, Sampaio E, Lucena E, Benone $S$. 2011. Prevalencia de mastitis bovina subclínica y microorganismos asociados: comparación entre ordeño manual y mecánico en Pernambuco, Brasil. Rev Salud Anim 33: 57-64.

33. Sanford CJ, Keefe GP, Sanchez J, Dingwell RT, Barkema HW, Leslie KE, Dohoo IR. 2006. Test characteristics from latent-class models of the California Mastitis Test. Prev Vet Med 77: 96-108.

34. Schalm O, Noorlander D. 1957. Experiments and observations leading to the development of California mastitis test. J Am Vet Med Assoc 130: 199-204.

35. Searcy R, Reyes O, Guajardo G 1995. Control of subclinical bovine mastitis: Utilization of a homoeopathic_combination. Brit Homoeopath J 84: 67-70. doi: 10.1016/S0007-0785(95)80033-6

36. Sharif A, Muhammad G. 2008. Somatic cell count as an indicator of udder health status under modern dairy production: A review. Pakistan Vet J 28: 194-200.

37. Solís M. 2007. Utilización de la solución hipertónica (agua de mar) en el tratamiento de la mastitis bovina en la finca «Guadalupana», del municipio de Nagarote, departamento de León. Tesis de Médico Veterinario. Nicaragua: Universidad Nacional Agraria. $110 \mathrm{p}$.

38. Torrez F, Duartes O. 2006. Estudio preliminar de la utilización de la manteca de armadillo (Dasypus novemcinctus) en el tratamiento de la mastitis bovina en el municipio de Paiwas, departamento de región autónoma del Atlántico Norte (RAAN). Tesis de Médico Veterinario. Nicaragua: Universidad Nacional Agraria. $55 \mathrm{p}$.

39. Zambrano JC, Echeverri J, LópezHerrera A. 2011. Alelos del gen BoLA DRB3.2 están asociados con mastitis en vacas lecheras. Rev Colomb Cienc Pecu 24: 145-156. 